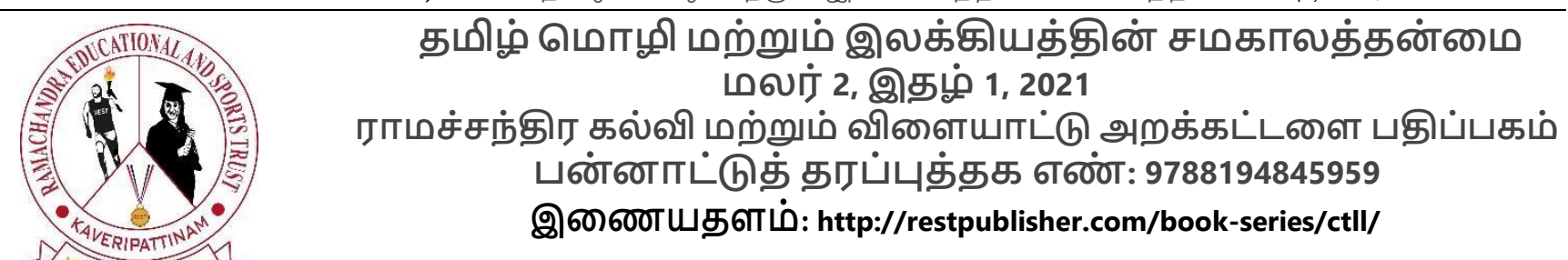

\title{
மூன்று படிகள் எழுதுதல்: ஆய்வுக் கட்டுரை எழுதும் வழிமுறை குறித்த வரைவு மா. பரமசிவன்
}

தமிழ் இலக்கியத்துறை, அருள் ஆனந்தர் கல்லூரி, கருமாத்தூர், மதுரை-625 514

ஆய்வைத் தொடங்குகின்ற ஆய்வாளர்களுக்கு எழுதுதல் என்பது சவாலான ஒன்று. சிலர், ‘ஆய்வு தொடர்பாக நாம் சிந்தித்துக் கொண்டு தானே இருக்கிறோம். எழுதிவிடலாம்’ என்று எண்னுவதுண்டு. வேறுசிலர், ‘நாளை எழுதுவோம். நாளை எழுதுவோம்' என்று நாட்களைத் தள்ளிப் போட்டுக் கொண்டே காலம் கடத்துவதும் உண்டு. இவ்வாறு நாட்களைத் தள்ளிப்போட்டுக் காலம் கடத்துவதை எழுத்தச்சம் (Blank Paper Fright)என்பர். இன்னும் சிலர், 'ஆய்வை அப்படி எழுதிவிடலாம்,இப்படி எழுதிவிடலாம்’ என்று கற்பனை செய்து கொண்டிருக்க, வேறுசிலர், ‘எழுதுவது எளிமையான செயல்' என்றும் கருதுவதுண்டு.ஆனால், எழுத முற்படும் போதுதான் அதில் இருக்கும் கடினம் ஆய்வாளருக்குப் புரியும்.

ஆய்வுக் கட்டுரை எழுதுதல் பொதுக்கட்டுரை எழுதுவதைப் போன்றதன்று. ஆய்வுக் கட்டுரையில் நாம் எழுதுகின்ற ஒவ்வொரு சொல்லுக்கும், பொருளும் சான்றுகளும் இருக்க வேண்டும். பொருளற்ற, சான்றுகளற்ற, காரண - காரிய இயைபுகளற்ற சொற்றொடர்கள் ஆய்விற்குப் பொருந்தாது. யூகத்தின் அடிப்படையில் கூறுதல் என்பதெல்லாம் பொதுக்கட்டுரைக்குப் பொருந்துமேயன்றி ஆய்வுக் கட்டுரைக்குப் பொருந்தாது. ஆய்வைப் பொறுத்தவரைச் சான்றுகளை உள்வாங்கி எழுதுதல் முதன்மையானதாகும். ஆய்வுக்கட்டுரை எழுதும் போது ஓர் ஆய்வாளர்ஆய்வு நடையைத் தான் பின்பற்ற வேண்டும். ஆய்வாளர் தன்னை முன்னிறுத்திக் கூறுகின்ற சொற்றொடர்களை முற்றிலுமாகத் தவிர்த்துக் கருத்தை முதன்மைப்படுத்தி, ஆய்வை முன்னிலைப்படுத்தும் விதமாகத் தமது ஆய்வுக் கட்டுரையை அமைக்க வேண்டும்.

\section{முதற்படி எழுதுதல் (First Draft)}

ஆய்வு எழுதுதலின் அடிப்படையே முதற்படி எழுதுதலில் தொடங்குகிறது. ஓர் ஆய்வாளர் எழுதுகின்ற முதல்படியை அப்படியின் மூன்றாம் படியுடன் ஒப்பிடும்போது அப்படிகள் இரண்டும் முற்றிலும் மாறி இருப்பதற்குக் கூட வாய்ப்பு உண்டு; பெரும்பாலான மாற்றம் இருப்பதே சரியானது.மூன்றாம் படியில் அந்தளவிற்கான கருத்துச் செறிவு ஏற்படுவதற்கும் முதற்படியே வழிகோலுகிறது. ஒரு ஆய்விற்கான முதற்படி எழுதுகின்ற ஆய்வாளர் ஆய்வுப் பகுதியின் கருத்துகளை உள்வாங்கி, தான் கூறவருகின்ற கருத்துக்கு முக்கியத்துவம் அளித்து எழுத வேண்டும். எந்தக் கருத்தை ஆய்வு வலியுறுத்த வேண்டுமோ, அந்தக் கருத்தை ஆய்வாளரின் சிந்தனைப் பின்புல அடிப்படையிலும் சான்றுகளை உள்வாங்கிக்கொண்டும் ஆய்வாளர் எழுதி முடிக்க வேண்டும்.முதற்படியை எழுதும் ஆய்வாளர் ஆய்வு தொடர்பான ஒரு சிறு குறிப்பைக் (Pullet tine Points) கையில் வைத்துக் கொண்டு எழுதுவது எந்தக் கருத்தையும் விட்டுவிடாமல் எழுதுவதற்கு வழிவகுக்கும்; சில சிறந்த கருத்துக்கள் தோன்றுவதற்கும் அடித்தளமாக அமையும்.

“குறிப்புகள் எடிக்காத நிலையில் எழுதப்படுவது சாதாரணக் கட்டுரையாக அமையுமே தவிர,

ஆய்வுக்கட்டுரையாக அமையாது (தமிழண்ணல் \& எம்.எஸ். இலக்குமணன், 2015: 96)"

என்ற கருத்தும் இங்குக் கவனத்தில் கொள்ளத்தக்கதாகும். அதே வேளை, ஆய்வைத் தொடங்கிய உடனே முதற்படி எழுதுதல் கூடாது. அது தொடர்பான தரவு சேகரிப்பு, தொடர்ந்த வாசிப்பு ஆகியன நிகழ்ந்தபின்னரேமுதற்படி எழுத வேண்டும். அப்பொழுதுதான் அம்முதற்படியில் ஆய்வு தொடர்பாக நமக்கு ஏற்பட்டிருக்கின்ற கருத்துப் புரிதல், தெளிவு ஆகியன வெளிப்படும்.

சிலர், ‘அனைத்துத் தரவுகளையும் தொகுத்த பிறகு எழுதத் தொடங்க வேண்டும்’ என்று எண்ணுவர். இது ஆய்வுக் கட்டுரை எழுதுவதற்குச் சரியான முறை என்றாலும், ஆய்வேடு உருவாக்கத்தைப் பொறுத்தவரைக் கால விரயத்தை ஏற்படுத்தும். ஆய்வேட்டின் ஒவ்வொரு கட்டுரைக்குமான தரவுகள் சேகரிக்கச் சேகரிக்க அந்தந்தக் பதிப்புரிமை: ராமச்சந்திர கல்வி மற்றும் விளையாட்டு அறக்கட்டளை 
கட்டுரைகளை ஒரு பக்கம் எழுதிக் கொண்டும் மற்றொரு பக்கம் பிற கட்டிரைகளுக்கான தரவுகளைச் சேகரித்துக் கொண்டுமான இரு செயல்பாடுகளும் இணைந்து நடைபெறுதல் வேண்டும்.அப்பொழுதுதான் குறிப்பிட்ட காலக்கட்டத்திற்குள் ஆய்வேட்டை நிறைவு செய்ய இயலும்.அதுமட்டுமன்றிக் கருத்திற்கு மட்டும் முதன்மை அளித்தால் போதுமானது. சந்திப்பிழை, எழுத்துப்பிழை முதலான இலக்கணப் பிழைகளுக்கும், தொடர்ப் பிழைகளுக்கும், நிறுத்தற் குறிகளுக்கும் முதன்மை அளிக்க வேண்டிய தேவையில்லை. இப் பிழைகளை அடுத்தடுத்த படிகளில் சாிசெய்து கொள்ளலாம். தாம் கூற வருகின்ற கருத்தை ஆற்றொழுக்காகக் கூறுகின்ற தன்மை முதற்படிக்குக் கட்டாயமாகும்.

ஆய்விற்கான முதற்படி எழுதும்போது,முதற்பக்கத்தோடு தொடர்புடைய ஒரு கருத்து மூன்று, நான்காம் பக்கங்கள் எழுதும்போது தோன்றினால், அதை உடனடியாக அந்தப் பக்கத்திலேயே தனிப்பத்தியாக எழுதிவிடுவது நல்லது. இரண்டாம் படி எழுதும் போது அதைக் கொண்டு வந்து முதற்பக்கக் கருத்தோடு சேர்த்துவிடலாம்.அதேபோல் முதற்பக்கத்தில் எழுதியதை மீண்டும் வேறொரு பக்கத்தில் எழுதும் சூழல் ஏற்படுமாயின், அதையும் தவிர்க்காமல் எழுதுவதே நல்லது.அவ்வாறு எழுதப்பெற்ற கருத்து எந்த இடத்திற்குப் பொருத்தமானது என்பதையும் இரண்டாம் படியில் சரி செய்து கொள்ளலாம்.அவ்வாறு செய்யாமல், ‘பிறகு எழுதுவோம்’ என்று எண்ணினால் அக்கருத்துகள் பின்னர் நினைவிற்கு வராமல் போய்விடிம்.

ஒரு கருத்தை நாம் எழுதிக் கொண்டிருக்கும் போதே அக்கட்டுரை தொடர்பான வேறு சில கருத்துகளும் தோன்றலாம்.அச்சூழலில், ஒரு கருத்திற்கு இடையில் வேறொரு கருத்தை எழுதுவதற்கு நெருடல் ஏற்பட்டால் ஏற்கனவே நாம் குறிப்பு வைத்திருக்கின்ற தாளில் நமக்குத் தோன்றும் குறிப்புகளைக் குறித்து வைத்துக் கொண்டு எழுதலாம். அந்தத் தாளில் இருக்கும் ஒவ்வொரு குறிப்புக் குறித்த தெளிவையும் எழுதி முடித்த பின்னர் அந்தக் குறிப்பை ( $\sqrt{ }$ ) செய்து கொள்வது நல்லது.

முதற்படியைப் பொறுத்தவரைத் தலைப்பிடல் என்பது ஒரு கருதுகோள் தன்மையில் அமையுமே தவிரத் தெளிவாக அமைந்து விடுவதில்லை. அவ்வாறு அமைக்கப்படும் தலைப்பு இரண்டாம் படி உருவாக்கத்தின்போது மாறலாம். இருப்பினும், தற்காலிகமாக ஒரு தலைப்பிட்டுஎழுதுவதே சிறந்தது. சில வேளைகளில் அந்தத் தற்காலிகத் தலைப்பே பொருத்தமானதாகவும் அமைந்து விடலாம்.ஆய்வுக் கட்டுரையில் நூற்பா, பாடலடிகளைப் பயன்படுத்தும் போது நமக்குத் தெரிந்தவரை அந்தப் பாடல் அடிகளை எழுதிக்கொண்டு, இரண்டாம் படி உருவாக்கத்தின் போது அதைச் செப்பனிட்டுக் கொள்வது நல்லது. அவ்வாறு செப்பனிடும் போது மிகுந்த கவனத்துடன் செயல்படுவது ஆய்வாளர் தம் கடமையாகும். பிறமொழிக் கலப்புடைய சொற்கள் முதல் படியில் வருவது தவறன்று. அதற்கு இணையான தூய தமிழ்ச் சொற்களை இரண்டாம்படி உருவாக்கத்தின் போது கட்டாயம் பயன்படுத்த வேண்டும்.அது தமிழ் தவிர்ந்த பிறமொழிக் கட்டுரை எனில் அந்தந்த மொழிகளுக்குரிய செம்மைப்படுத்தப்பெற்ற சொற்களை இரண்டாம் படியில் கவனத்துடன் பயன்படுத்துவது சிறந்ததாகும்.

முதற்படி எழுதுகின்ற ஆய்வாளர் முதற்பக்கத்தை எழுதி முடித்த பின்னர் உடனேயே அந்தப் பக்கத்தைத் திரும்பப் பார்ப்பதைத் தவிர்க்க வேண்டும். அவ்வாறு நாம் பார்க்கும்போது அதில் சில பிழைகள் தென்படலாம். அந்நிலையில், அதைத் திருத்துகின்ற மனநிலைதான் நமக்கு வருமே ஒழியத் தொடர்ச்சியாக எழுதுகின்ற மனநிலை தோன்றாது.எந்தத் திருத்தங்களாக இ இருந்தாலும் இரண்டு, மூன்றாம் படிநிலைகளில் செய்வதே பொருத்தமுடையதாகும்.முதற்படி எழுதும்போது பின்வரும் குறிக்கோள்களைக் கொண்டிருக்க வேண்டும் (தமிழண்ணல் \& எம்.எஸ். இலக்குமணன், 2015: 102). அவைவருமாறு:

1. மெய்மைச் சான்று முழுமையும் (Fullness of Facts)

2. மெய்மைகளின் சுருக்கமும் செம்மையும் (Precision and Accuracy of Facts)

3. மெய்மைகளின் காரண, காரிய இயைபு (Coherence or Logic facts)

4. மெய்மை, கருத்துகளின் வைப்புமுறை அல்லது கருத்தோட்டம் (Movement or trasition of Facts and Ideas).

இந்தக் குறிக்கோள்கள் முதற்படியில் முழுமை அடைந்திருக்கின்றனவா என்பதைச் சரிபார்த்தல் ஆய்வாளர் தம் கடமையாகும்.

முதற்படி என்பது, ‘ஒரு சிற்பக் கலைஞன் ஒரு சிற்பத்திற்கு அடி கோலுவதைப் போன்றது'. 
அந்தஅடிகோலுதல்சிறப்பாக அமையவில்லை எனில், சிற்பம் சிறப்பாக அமையாது. 'முதல் கோணல் முற்றிலும் கோணல்’ என்ற நிலையாகிவிடும். அதனால் கவனச்சிதைவு ஏதுமின்றி எழுத முயற்சிப்பது முக்கியமானதாகும். முதற்படி எழுதும் ஆய்வாளர் தம் குறிக்கோள் நிலையில் கவனமாக இருத்தல் மட்டுமின்றிப் புறச்சூழலிலும் கவனம் செலுத்த வேண்டும். முதற்படி எழுதும்போது கவனத்தில் கொள்ள வேண்டிய புறச்சூழல் தன்மைகளைப் பின்வருமாறு வரிசைப்படுத்தலாம்.

1. முதற்படியை அதிகாலையில் எழுத முயற்சிப்பது நலம் பயக்கும். இரவு முழுவதும் நாம் உறங்கும் போது நமது மூளைக்குத் தேவையான ஓய்வு கிடைத்து விடுகிறது. அதிகாலையில் புறச்சூழலும் அமைதியாக இருக்கும். இந்நிலையில், மூளைக்கு எந்தவிதமான தயக்கமும் புறவயத்தாக்கமும் இருக்காது. இச்சூழலில், முதற்படி எழுதுதல் கருத்தாழமிக்க ஆய்வுக்கட்டுரை உருவாக வழிவகுக்கும். அதேவேளை, இரவு உறங்கச் செல்லும்போது காலை எழுத இருக்கின்ற ஆய்வுக்கட்டுரை தொடர்பாகச் சிந்தித்துவிட்டு, ஒரு சில குறிப்புகளை எழுதி வைத்து விட்டு உறங்கச் செல்வது நல்லது .

2. அதிகாலை எழுந்திருக்கும் பழக்கம் இல்லாதவர் வழக்கம்போல் காலையில் எழுந்தவுடன் எழுதுவது நல்லது . என்னதான் நண்பகல், மாலை, இரவு வேளைகளில் எழுதினாலும், மூளைக்கு ஓய்வளித்து உறங்கி எழுந்தவுடன் எழுதும்போது தோன்றுகின்ற கருத்தாழம் பிற நேரங்களில் எழுதும் எழுத்தில் கிடைக்காது . இதைக் கவனத்தில் கொள்வது நலம் பயக்கும்.

3. ஒருவேளை காலையில் எழுத முடியவே இல்லை எனும் சூழல் ஏற்பட்டால், பகலில் எழுத முற்படும்போது அரைமணிநேர உறக்கத்திற்குப் பின்னர் அமைதியான சூழலில்எழுத முற்படுவது கருத்தாழமிக்க முதற்படி தோன்றுவதற்கு வழிவகுக்கும்.

4. அதிகாலையில் முதற்படி எழுதுவதற்காக நாம் எழுந்திருக்கும் போது எழுதுகோல் (Pen and Pencil), தாள் முதலான எந்தப் பொருட்களையும் தேடிக் கொண்டிருக்க கூடாது. அப்படித் தேடும்போது சில பொருட்கள் உடனே கிடைக்காத சூழலில் மூளை சோர்வடைய வாய்ப்பு உண்டு. அதனால் முதற்படி நாம் கருதுகின்ற அளவிற்கு ஆழமாக வருவதற்கும் வாய்ப்பு இல்லாமல் போய் விடிம். எனவே, முதல் நாள் இரவில் நமக்குத் தேவையான அனைத்துப் பொருட்களையும் எடுத்து வைத்துவிட்டுக் காலையில் எழுந்தவுடன் எதையும் தேடிக் காலம் கடத்தி மூளைக்குச் சோர்வளிக்காமல் எழுதத் தொடங்க வேண்டும் .

5. அதிகாலையில் எழுதுகின்ற பழக்கமுடையவர்கள் கவனத்தில் கொள்ள வேண்டிய மற்றொரு புறச்சூழலும் உண்டு. அதிகாலையில் நாம் எழுதத் தொடங்கும் போதோ அல்லது எழுதிக்கொண்டிருக்கும் போதோ மின்சாரம் நின்று விடலாம். அப்போதும் மூளை சோர்வடைய வாய்ப்புண்டு. அதனால் முதல் நாள் இரவில் எழுதுகோல் முதலான பொருட்களை எடுத்து வைக்கும் போதே நமக்குத் தேவையான வெளிச்சத்தை தருகின்ற மெழுகுவர்த்தி (Handle), கைவிளக்கு (Emergency Light) முதலானவற்றையும் எடுத்து வைத்துக்கொள்வது முக்கியமானதாகும்.

6. முதற்படி எழுதும் போது கைபேசியை முற்றிலும் தவிர்த்தல் நல்லது.

7. முதற்படி எழுதுகின்ற ஆய்வாளர் எந்தச் சூழலிலும் புறவயத்தாக்கம் இல்லாமல் பார்த்துக் கொள்வது இன்றியமையாததாகும்.

8. முதற்படி எழுதும் ஆய்வாளர்ஆய்வுக்கட்டுரை முழுவதையும் ஒரே அமர்வில் எழுதிவிட வேண்டும். முதற்பாதியை ஒரு அமர்விலும் அடுத்தபாதியை அடுத்த அமர்விலும் எழுதும்போது அது கருத்துச் சிதைவை ஏற்படுத்த வாய்ப்பு உண்டு.

மேற்கண்டவாறு புறக்காரணிகளிலும் நாம் கவனம் செலுத்தி முதல் படியை எழுதும்போது அம்முதற்படி சிறந்த ஆய்வுத்தரம் மிகுந்ததாக உருவாகும்.

\section{இரண்டாம் படி எழுதுதல் (Second Draft)}

‘ஒரு சிற்பி ஒரு சிற்பத்திற்கு அடிகோலுவதைப் போன்றது முதற்படி. என்றால், அந்தச் சிற்பத்தைச் செப்பனிடுவதைப் போன்றது இரண்டாம் படி.' இரண்டாம் படியில் தான் ஆய்வுக்கட்டுமை முழுமை அமையும் நிலைக்கு வருகிறது. முதற்படி நிறைவு பெற்றவுடன் ஆய்வுக் கட்டுரையின் இரண்டாம் படிக்கான பணியை உடனே செய்து விடக்கூடாது. குறைந்தது ஒரு நாளாவது கழித்துத்தான் இரண்டாம் படிக்குாிய திருத்தத்தை மேற்கொள்ள வேண்டும். அச்சூழலில், நாம் எழுதிய ஆய்வுக்கட்டுரை தொடர்பான சிந்தனை நம் மனதில் அடிக்கடி ஓடிக்கொண்டே 
இருக்கவேண்டும். அக்கட்டுரை தொடர்பாக நாம் சேகரித்திருக்கின்ற தரவுகள், குறிப்புகள் இவை அனைத்தையும் சரி பார்த்துக் கொண்டு முதற்படியைத் திருத்தும் பணிக்குச் செல்வது ஆய்வுக்கட்டுரை செப்பமடைவதற்கு வழிவகுக்கும்.

முதற்படியை நாம் திருத்தும்போது முதற்படி எழுதியதிலிருந்து வேறுபட்ட நிறமுடைய எழுதுகோலைப் பயன்படுத்த வேண்டும். அப்போதுதான், அதன்மீது நாம் திருத்துகின்ற இரண்டாம் படிக்கான வேறுபாடுகள் தெரியும். முதற்படியை, ஒரு கருத்துருவாக்கத்தின் அடிப்படையில் திருத்தும் சூழலில், அதைவிடச் சிறந்த வேறொரு கருத்துருவாக்கம் தோன்றினால், மூன்றாவது நிறமுடைய பேனாவால் அக்கருத்துருவாக்கத்தைஎழுத வேண்டும்.படி எடுக்கும் போது அக்கருத்துருவாக்கங்களில் சிறந்தவற்றைப் படி எடுத்துக் கொள்ளலாம்.

கூடுதல் விளக்கம் அளித்தல், சான்று விளக்கத்தைச் சேர்த்தல், மொழிநடையைச் சாிசெய்தல், சந்திப்பிழை ஒருமைப் பன்மைப் பிழை முதலான தொடர்ப்பிழைகளைச் செப்பனிடிதல், சொல்லாட்சித் திறன், தொடராட்சித் திறன்களை மேம்படுத்துதல், பத்தி பிரித்தல், பாடலடிகளைச் சரிசெய்தல், பாடலடிகளின் அடி எண்களைச் சாியாகக் குறித்தல், ஆய்வுக் கட்டுரையின் இடையே வந்துள்ள பிறமொழிச் சொற்களைச் சாிசெய்தல், சான்றெண் விளக்கம் அடிக்குறிப்பெண்களைச் சரிசெய்தல், ஒற்றை மேற்கோள் - இரட்டை மேற்கோள் முதலான அனைத்து வகையான நிறுத்தற் குறிகளையும் ஒழுங்குபடுத்துதல், மேற்கோள்களை உள்ளடக்கியோ (Indent) அல்லது சாய்வு எழுத்தில் (Italic) அமைப்பதையோ தெளிவுபடுத்துதல் ஆகிய அனைத்துப் பணிகளும் இந்த இரண்டாம் படி உருவாக்கத்திற்கான முதற்படித் திருத்தத்தில்தான் மேற்கொள்ளவேண்டும்.

இவை மட்டுமன்றி உட்தலைப்புகள் அளித்தல், தேவையற்ற உட்தலைப்புகளை நீக்குதல், முதன்மைத் தலைப்பைச் செப்பனிடல் ஆகிய பணிகளையும் முதல்படித் திருத்தத்தில்தான் மேற்கொள்ள வேண்டும். ஆய்வுக்கட்டுரையின் முன்னுரை, முடிவுரைகளில் கூடுதல் கவனம் செலுத்துதலும் இந்தப் படியின் முதன்மைப் பணியாகும். மேலும், ஆய்வுக் கட்டிரையின் தெளிவிற்குத் தேவைப்படுகின்ற அட்டவணைகள் (Tables), வரைபடங்கள் (Flow charts), நிலவரைபடம் (Maps), ஒளிப்படங்கள் (Photos) முதலானவற்றையும் அவை குறித்த விளக்கங்களைச் சரிசெய்தலும் இவ்வரைவில் மேற்கொள்ள வேண்டிய முக்கியப் பணிகளாகும்.

நான் முதல் வரைவை எழுதிக் கொண்டே போவேன். சிறிது மாற்றக் கருதித் தொடங்குவேன். பெரிய

அளவுக்கு மாற்றங்கள் நேரும். தாளில் மூன்றாவது உலகப் போர் நிகழ்ந்தது போல அடித்தல்,

திருத்தல்கள் காணப்படும் (மேற்கோள், 2009: 211)

என்ற வைரமுத்துவின் கருத்தும் இங்கு நினைவில்கொள்ளத்தக்கதாகும். முதற்படியைத் திருத்திய பின்னர் ‘இம்முதல் படி நாம் எழுதியதுதானா?' என்ற வினா எழும் அளவிற்குக் கருத்துச் செறிவாலும் மொழி நடையாலும் வளர்ச்சியடைந்திருக்குமாறு திருத்த வேண்டும். இந்தத் திருத்தத்தை நெருடல் ஏதுமின்றித் தாராளமாக, முழுச் சுதந்திரத்தோடு, கருத்துப் புரிதலின் அடிப்படையில் மேற்கொள்ள வேண்டும்.

இப்பணிகள் அனைத்தையும் சரிசெய்த பின்னர் முதல் படியின் மீது திருத்தப் பெற்றிருக்கின்ற அப்படியைப் படியெடுத்தல் தான் இரண்டாம் படி ஆகும். இவ் இரண்டாம் படி உருவாக்கத்தின்போது ஆய்வாளர்கள் கவனத்தில் கொள்ள வேண்டியனவாகப் பின்வருவனவற்றைக் கூறலாம்.

1. சுருக்கம், தெளிவுடன் இருத்தல்.

2. தேவையான கருத்துகளை வலியுறுத்தல்.

3. எளிய ஆய்வு நடையில் இருத்தல்; மிகக் கடினமான கலவை வாக்கியங்களைத் தவிர்த்தல்.

4. கூறியது கூறல், மயக்கம் தருதல் போன்ற சொற்களைத் தவிர்த்தல்.

5. சொல்லாட்சித் திறனை மேம்படுத்துதல்.

இக்கருத்துகள் மட்டுமின்றி, ‘இரண்டாம் படியை நிறைவு செய்த பின்னர் சில தெரிவினாக்களை அந்தப் படியின் மீது பொருத்திப் பார்த்த வேண்டும் என்றும் அத்தெரிவினாக்கள்இரண்டாம் படியின் தெளிவைப் புலப்படுத்தும்’ என்றும் அறிஞர்கள் கருதுகின்றனர் (தமிழண்ணல் \& எம்.எஸ். இலக்குமணன், 2015: 109-111). அத்தெரிவினாக்கள் வருமாறு:

1. ஆய்வேட்டுத் தலைப்பு, ஆய்வுப்பொருள் முழுவதையும் உள்ளிட்டதுதானா?

2. தொடக்கத்தில் மேற்கொண்ட யூகம் அல்லது கருதுகோள் போதுமான அளவு சோதனை செய்து ஆராயப்பட்டதா? 
3. ஆய்வறிக்கையின் உட்பிரிவுகளும் தலைப்புகளும் காரண காரிய முறையில் அமைந்துள்ளனவா?

4. மெய்ம்மைகள்போதுமான அளவு தொகுக்கப்பட்டனவா? தவறின்றி விளக்கப்பட்டனவா?

5. கண்டுபிடிப்புகள், உய்த்துணர்வுகள், முடிபுகள் யாவும் மெய்ம்மைகளின் அடிப்படையிலேயே பெறப்பட்டு வெளிப்படிகின்றனவா?

6. ஆய்வேட்டின் தொடக்கம் முன்னுரையாகவும் முடிவு முடிவுரையாகவும் அமைந்து காணப்படுகின்றனவா?

7. தொடக்கவுரை முதல் பரிந்துரை ஈறாக ஆற்றொழுக்குப் போன்ற வளர்ச்சியுளதா?

8. போதிய அளவு சிந்தனை செலுத்தப்பட்டுள்ளதா? அச்சிந்தனை தெளிவாயுளதா? அது தருக்க நெறியினதாகவும் செம்மையாகவும் முழுமையாகவும் உளதா?

9. ஆய்வறிக்கை எந்த அளவு முடியுமோ அந்த அளவிற்கு முழுவதும் செம்மையாகவுளதா?

10. முடிபுகள் அழுத்தமாகக் கூறப்பட்டு, ஆய்வறிக்கையின் வலுவுள்ள பகுதியாக அப்பகுதி விளங்குகிறதா?

11. ஆய்வறிக்கை ஒரு செய்தியை அறிவிக்கும் அடிக்கருத்துடையதாய் ஒருமைப்பாட்டுடன் திகழ்கிறதா? அல்லது உதிரியான கருத்துகளின் தொகுதியாக விளங்குகிறதா? சிதறிக் கிடக்கும் மெய்ம்மைகள் போல் உளதா?

12. தொடக்கத்தொடர், பத்திப் பிரிவு போல்வன உள்ளத்தைக் கவரும்படி அமைந்துள்ளனவா?

13. பத்திகள், உட்பிரிவுகள், இயல்கள் அனைத்தும் ஒருங்கினைந்து ஆய்வறிக்கை முழுமையாகவும் அளவோடும் வைப்பு முறையோடிம் அமைந்துள்ளதா?

14. ஒவ்வொரு பத்தியும் அல்லது உட்பிரிவும் பயனுடையதாக ஒரு கருத்தோடு அமைந்ததா?

15. ஒவ்வொரு வாக்கியமும் தன்னளவில் நிலைபேறுடையதா? அல்லது பல்வேறு சார்புகளாலும் காலங்கடந்த சிந்தனைகளாலுமே அவை நிலைபெறுகின்றனவா?

16. நடை எளிமையாய், ஆற்றொழுக்காய் உளதா? சொல்லும் தொடரும் இயல்பாகவும் மரபுப் பண்போடும் அமைந்துளவா?

17. வெளிப்படுத்துதலும் எழுத்து முறையும் ஒரு கருத்தைத் கருதித் தோன்றி வலுவுடன் விளங்குகின்றனவா? அதை ஆற்றலோடும் நாகரிகமாகவும் வெளியிடுகின்றனவா?

18. கருத்துக்கள், சொற்கள், தொடர்கள் ஆகியன தம்முள் இயைந்து நின்று ஒழுங்கு முறைப்படியும் நிரலாகவும் வருமாறு (வைப்பு முறை) பின்பற்றப்பட்டுள்ளதா?

19. ஆய்வறிக்கைப் போக்கு மிகக்கடினமாய், ஆய்வாளரின் உயர்கல்விப் படிப்பைக் காட்டுவதாய் உளதா? மிக எளிதாய் அனைத்தும் அறியப்பட்டதொன்றாய்க் காணப்படுகிறதா?

20. படிப்பவர் பற்றி மனக்கருத்திற் கொண்டு எழுதப்பட்டதாகத் தோன்றுகிறதா? ஆய்வாளர் வாசகரை நினையாமல் தமக்காகவே எழுதியது போல் உளதா?

21. ஒவ்வொரு சொல்லும் நிறுத்திப் பார்க்கப்பட்டு அதன் உண்மைப் பொருளை அறிந்து பயன்படுத்தப்பட்டுள்ளதா? அதன் ஒரு குறிப்பிட்ட செயலை நிறைவேற்ற அது ஆளப்பட்டுள்ளதா?

22. எழுத்து நடை தீர்மானமற்ற ஒருவேளை இருந்த போதிலும், கூடும் என்பன போன்ற வாசகங்களை மிக அதிகமாகக் கொண்டு, பல இடங்களில் இவ்வாறு முடிபற்ற நிலைகளையே தோற்றுகிறதா?

23. எழுத்துநடை மிக அதிகமாக - மிகையாகக் கூறப்பட்ட - ‘மிகவும்',உறுதியாக’, ‘மறுப்பின்றி', ‘ஆணித்தரமாக’ என்பன போன்ற வலியுறுத்து முறைகளைக் கொண்டு விளங்குகிறதா?

24. திரிசொற்களும் தெளிவற்ற சொற்களும் அளவுக்கு மேல் பயன்படுத்தப் பட்டுள்ளனவா?

25. ஆய்வாளர் தமக்குத் தாமே திறனாளியாய்ப் பார்க்கும் போது தம் ஆய்வேடு தமக்கு மனநிறைவு தருவதாக உணர்கிறாரா?

இந்த வினாக்கள் அனைத்திற்குமான விடை நாம் எழுதியுள்ள இரண்டாம் படியில் இருக்குமாயின் நாம் தெளிவுறுத்த விழைகின்ற ஆய்வுச் சிக்கலைத் தெளிவுபடுத்தியுள்ளோம் என்பது பொருளாகும்.

\section{மூன்றாம் படி எழுதுதல் (Third Draft)}


இரண்டாம் படியை மீண்டும் ஒரு நாள் அல்லது ஓரிரு நாட்கள் கழித்து வாசிக்கும்போது சிற்சில சொற்றொடர் மாற்றங்கள், ஒற்றுப்பிழை, எழுத்துப்பிழைகள் முதலானவை கண்னுக்குப் புலப்பட வாய்ப்பு உண்டு. அவற்றைச் சரிசெய்து அதைப் படியெடுத்துக் கொள்ளவேண்டும். அதுவே, மூன்றாம் படியாகும். மூன்றாம் படி என்பது பெரும்பாலும் திருத்தங்கள் ஏதுமின்றிப் படியெடுத்தலாகவே அமையும்; அமைய வேண்டும்.

ஓர் ஆய்வுக்கட்டுரையைப் பொறுத்தவரை மூன்று படிகள் மட்டும் போதுமானது என்பதில்லை. மூன்று படிகள் எழுதிய உடனே நெறியாளரிடம் அளிக்க வேண்டும் என்ற கட்டாயமுமில்லை. மூன்று படிகளாவது எழுத வேண்டும் என்பதுதான் இதன் முதன்மை நோக்கமாகும். மூன்று படிகள் எழுதி முடித்தவுடன் ஆய்வுப் பழக்கமுள்ள, ஆய்வாளருக்கு நெருக்கமான,நடுநிலையோடு சிந்திக்கக் கூடிய, துறைசார்ந்த ஆய்வாளர் ஓரிருவரிடம் தமது ஆய்வுக் கட்டுரையை அளித்து, வாசிக்கச் செய்து, திருத்தங்களைக் குறிக்கச் செய்யலாம். அந்தத் திருத்தங்களையும் சரிசெய்த பின்னர் ஆய்வு நெறியாளரிடம் அளிக்கும் போது அந்த ஆய்வுக் கட்டுரை மேலும் தெளிவுபெறும்.அத்தெளிவுகளுடன்ஆய்வு நெறியாளர் செய்கின்ற திருத்தங்களும், ஆய்வாளர் எழுதுகின்ற ஆய்வுக் கட்டுரைகளின் தரம் உயர்வதற்கு வழிவகுக்கும்.அதன் பின்னர் தான் ஆய்வுக் கட்டுரையைக் கணினியாக்கத்திற்கு அளிக்க வேண்டும் .

இன்றைய தொழில்நுட்ப உலகில் கைபேசியைக் கையில் வைத்துக்கொண்டு பேசினாலே கணினியாக்கப் பெறுகின்ற மென்பொருள்கள் எல்லாம் புழக்கத்திற்கு வந்து விட்டன. இதனால்,ஆய்வாளர்கள் சிலர் ஆய்வின் முதற்படியை அந்த மென்பொருளைப் பயன்படுத்திப் பேசிக் கணினியாக்கம் செய்து, அதன் பின்னர் அதை ஒட்டிய திருத்தங்களை மேற்கொள்கின்றனர். அது முற்றிலும் தவிர்க்கப்பட வேண்டிய செயலாகும்.‘ஆய்விற்குத்தான் கணினியாக்கமே அன்றிக் கணினியாக்கத்திற்காக ஆய்வன்று' என்பதை இன்றைய ஆய்வாளர்கள் கவனத்தில் கொள்ளுதல் வேண்டும். மூன்று படிகளும் எழுதி, துறை சார்ந்த ஓரிருவரை வாசிக்கச் செய்து, ஆய்வு நெறியாளரிடம் அளித்துத் திருத்தங்களை மேற்கொண்ட பின்னரே ஆய்வுக்கட்டுரையைக்(கைபேசி வழியாகவோ அல்லது கணினி வழியாகவோ) கணினியாக்கம் செய்ய வேண்டும். இதைவிடுத்துக் கணினியாக்கம் செய்த பின்னர் திருத்தங்களை மேற்கொள்வது தவிர்க்கப்பட வேண்டிய செயலாகும்.

முதல் மூன்று படிகள் கையெழுத்தில் எழுதப்பெறாமல், கைப்படத் திருத்தம் பெறாமல் கைபேசி வழிக் கணினியாக்கம் செய்வது தற்செயல் கருத்துப் பதிவுகளாக அமைந்து விடுமேயன்றித் தரமான ஆய்வுக் கருத்துகளாக அமையாது. மேலும், கணினியாக்கம் செய்ததைத் திருத்துவதற்கான மனக்கூச்சம் ஏற்படவும் வாய்ப்புண்டு.அப்போது அது கருத்துச் செறிவையும் தடை செய்துவிடும். இதுமட்டுமன்றிச் செம்மையான சொற்பயன்பாடு குறைவதற்கு வாய்ப்புண்டு என்பதையும் கவனத்தில் கொள்ளுதல் வேண்டும்.

மூன்று படிகள் என்பதைவிட நான்கு, ஐந்து படிகள் எழுதும்போதுதான் ஆய்வுக்கட்டுரை மிகத்தரமானதாக உருவாகும். ஆய்வுக் கட்டுரை எழுதும் ஆய்வாளர்கள் மூன்று படிகளாவது கட்டாயம் எழுத வேண்டும் என்பதுஇங்குக் கவனத்தில் கொள்ளத்தக்கதாகும்.

\section{துணை நின்றவை}

- தமிழண்ணல் \& இலக்குமணன் எம்.எஸ், 2015 (16ஆம் ப.), ஆய்வியல் அறிமுகம்,மதுரை: செல்லப்பா பதிப்பகம்.

- பாலசுப்பிரமணியன் கு.வெ., 2009 (நா.ப.), ஆய்வியல் நெறிகள், தஞ்சாவூர்: உமா நூல் வெளியீட்டகம்.

- முத்துச்சண்முகன் வேங்கடராமன் சு., 2013, இலீக்கிய ஆராய்ச்சி நெறிமுறைகள்,சென்னை: நியூ செஞ்சுரி புத்தக நிலையம். 